\title{
BMJ Open Hypoglycaemia Awareness Restoration Programme for People with Type 1 Diabetes and Problematic Hypoglycaemia Persisting Despite Optimised Self-care (HARPdoc): protocol for a group randomised controlled trial of a novel intervention addressing cognitions
}

\author{
Stephanie A Amiel, ${ }^{01,2}$ Pratik Choudhary, ${ }^{1}$ Peter Jacob, ${ }^{1}$ Emma Lauretta Smith, ${ }^{2}$ \\ Nicole De Zoysa, ${ }^{2}$ Linda Gonder-Frederick, ${ }^{3}$ Mike Kendall, ${ }^{1}$ Simon Heller, ${ }^{4}$ \\ Augustin Brooks, ${ }^{5}$ Elena Toschi, ${ }^{6}$ Dulmini Kariyawasam, ${ }^{7}$ Laura Potts, ${ }^{8}$ Andy Healy, ${ }^{8}$ \\ Helen Rogers, ${ }^{2}$ Nick Sevdalis, ${ }^{9}$ Marietta Stadler, ${ }^{1}$ Mustabshira Qayyum, ${ }^{1}$ \\ Ioannis Bakolis, ${ }^{8}$ Kimberley Goldsmith ${ }^{8}$
}

To cite: Amiel SA, Choudhary P, Jacob P, et al. Hypoglycaemia Awareness Restoration Programme for People with Type 1 Diabetes and Problematic Hypoglycaemia Persisting Despite 0ptimised Self-care (HARPdoc): protocol for a group randomised controlled trial of a novel intervention addressing cognitions. BMJ Open 2019;9:e030356. doi:10.1136/ bmjopen-2019-030356

- Prepublication history and additional material for this paper are available online. To view please visit the journal (http:// dx.doi.org/10.1136/bmjopen2019-030356).

Received 10 March 2019 Revised 10 April 2019 Accepted 12 April 2019

Check for updates

(C) Author(s) (or their employer(s)) 2019. Re-use permitted under CC BY-NC. No commercial re-use. See rights and permissions. Published by BMJ.

For numbered affiliations see end of article.

Correspondence to

Professor Stephanie A Amiel; stephanie.amiel@kcl.ac.uk

\section{ABSTRACT}

Introduction Severe hypoglycaemia (SH), when blood glucose falls too low to support brain function, is the most feared acute complication of insulin therapy for type 1 diabetes mellitus (T1DM). $10 \%$ of people with T1DM contribute nearly $70 \%$ of all episodes, with impaired awareness of hypoglycaemia (IAH) a major risk factor. People with IAH may be refractory to conventional approaches to reduce $\mathrm{SH}$, with evidence for cognitive barriers to hypoglycaemia avoidance. This paper describes the protocol for the Hypoglycaemia Awareness Restoration Programme for People with Type 1 Diabetes and Problematic Hypoglycaemia Persisting Despite Optimised Self-care (HARPdoc) study, a trial to assess the impact on hypoglycaemia experience of a novel intervention that addresses cognitive barriers to hypoglycaemia avoidance, compared with an existing control intervention, recommended by the National Institute of Health and Care Excellence.

Methods and analysis A randomised parallel twoarm trial of two group therapies: HARPdoc versus Blood Glucose Awareness Training, among 96 adults with T1DM and problematic hypoglycaemia, despite attendance at education with or without technology use, in four centres providing specialist T1DM services. The primary outcome will be the SH rate at 12 and/or 24 months after randomisation to either course. Secondary outcomes include rates of $\mathrm{SH}$ requiring parenteral therapy, involving unconsciousness or needing emergency services; hypoglycaemia awareness status, overall diabetes control and quality of life measures. An implementation study to evaluate how the interventions are delivered and how implementation impacts on clinical effectiveness is planned as a parallel study, with its own protocol.

\section{Strengths and limitations of this study}

- A randomised trial of two active interventions, one novel and one established, for a very vulnerable group of people, failing to achieve safety using conventional management strategies, which themselves have been subject to a systematic review.

- The trial targets severe hypoglycaemia in adults with type 1 diabetes, a major challenge for people with the condition, their families and the healthcare systems supporting them.

- The novel intervention addresses cognitions, identified as barriers to behaviour change, in the maintenance of behaviours that create high risk of continuing to experience severe hypoglycaemia, while the comparator intervention has not previously been tested in the National Health Service.

- The study is run in four specialist diabetes services, in the UK and the USA, and is supported in its delivery by a certified clinical trial unit.

- The study requires correct identification of people whose hypoglycaemia problem has remained refractory to conventional management.

Ethics and dissemination The protocol was approved by the London Dulwich Research Ethics Committee, the Health Research Authority, National Health Service R\&D and the Institutional Review Board of the Joslin Diabetes Center in the USA. Study findings will be disseminated to study participants and through peer-reviewed publications and conference presentations, including user groups. Trial registration number NCY02940873; Pre-results. 


\section{INTRODUCTION}

Severe hypoglycaemia (SH) is the most feared acute complication of insulin therapy for insulin deficient (type 1 and late type 2) diabetes. ${ }^{1} \mathrm{SH}$ occurs when plasma glucose concentrations fall so low that the person experiencing the hypoglycaemia is rendered incapable of self-treatment and/or experiences loss of consciousness or seizure. ${ }^{2} \mathrm{SH}$ causes confusion, abnormal behaviour, family trauma, restricted work opportunities ${ }^{3}{ }^{4}$ and sudden cardiac death. ${ }^{5}$ Each year, about $40 \%$ of people with type 1 diabetes mellitus (T1DM) will experience SH. ${ }^{6}$ Four to $10 \%$ of deaths in adults with T1DM under the age of 40 years may be due to $\mathrm{SH}^{7-10}$ with $550-1391$ deaths attributed to it annually in England and Wales. ${ }^{11}$ Fear of hypoglycaemia can prevent people with diabetes achieving the glucose control associated with reduced risk of long-term vascular complications of diabetes, ${ }^{1}$ which can affect the eyes, kidneys and peripheral nerves and drive premature cardiovascular and cerebrovascular disease. $^{12}$

People with T1DM have defects in endogenous counter-regulatory mechanisms that maintain plasma glucose. ${ }^{13} 14$ Their best protection against $\mathrm{SH}$ is subjective awareness of the start of a plasma glucose fall, driving ingestion of rapidly absorbed carbohydrate. Impaired awareness of hypoglycaemia (IAH) is a major predictors of $\mathrm{SH}^{6}{ }^{6}$ increasing risk of SH sixfold to eightfold in T1DM. ${ }^{1516}$. IAH affects $25 \%-40 \%$ of people with T1DM of $15+$ years' duration..$^{15} 16$ IAH impairs quality of life, causing loss of privileges such as licence to drive and problems at home and at work. ${ }^{4} 1718$

IAH is associated with defects in the subjective and counter-regulatory neuroendocrine responses to hypoglycaemia, additional to the inability to regulate insulin and glucagon concentrations in response to hypoglycaemia of T1DM. ${ }^{19}$ These defects are inducible by exposure to hypoglycaemia ${ }^{2021}$ and reversible by avoidance of plasma glucose $<3 \mathrm{mmol} / \mathrm{L} \quad(<54 \mathrm{mg} / \mathrm{dL}){ }^{22-24}$ Hypoglycaemia awareness and/or reduced risk of SH can be restored in clinical settings. A meta-analysis of structured education in flexible insulin therapy, aiming to transfer skills of insulin dose adjustment to users, or to enhance people's subjective ability to detect or predict extremes of plasma glucose and avoid them, showed significant reductions in $\mathrm{SH}^{25}$ with additional evidence from the comparison of optimised MDI (multiple daily insulin injections) vs pumps with or without sensors in severe hypoglycaemia (HypoCOMPaSS) trial. ${ }^{26}$ Two programmes, the UK's Dose Adjustment for Normal Eating (DAFNE) and the US programme Blood Glucose Awareness Training $(\text { BGAT })^{27-29}$ have also been shown to restore awareness of hypoglycaemia to some. Forty-three per cent of those entering DAFNE with IAH had recovered awareness at 1 year, patients then reporting IAH contributing almost all the residual $\mathrm{SH}$ in the programme. ${ }^{16}$ Newer technologies for insulin delivery (continuous subcutaneous insulin infusion, CSII) and continuous glucose monitoring (CGM) and sensor augmented pump therapy can also reduce $\mathrm{SH}^{30-33}$ However, there are no data on the impact of CSII on IAH, and use of real-time CGM does not restore IAH, making patients dependent on the technology longterm. ${ }^{34}$ Guidelines suggest a stepwise approach through education and technology, but some people continue to report IAH and $\mathrm{SH}^{16}$; some cannot engage successfully with technological interventions. ${ }^{35}$ Anecdotally, people gaining protection from $\mathrm{SH}$ using CGM experience $\mathrm{SH}$ when not wearing the devices. As the last resort, beta-cell replacement by islet or pancreas transplantation is an option ${ }^{36}$ but is costly, requires long-term immunosuppression and is not universally available or acceptable. Additional interventions are urgently needed.

Some people with IAH show unexpectedly low concern about the consequences of their hypoglycaemia experience and describe beliefs about their hypoglycaemia that may be barriers to future hypoglycaemia avoidance. ${ }^{3738} \mathrm{In}$ a Swedish study, $8 \%$ of people were at high risk of SH but had low concern about it. ${ }^{39}$ In a UK clinic audit, people with IAH were less likely to use strategies decided on in clinic consultations than people with hypoglycaemia awareness. ${ }^{40}$

Based on the above evidence, we hypothesised that an intervention that directly addresses the described cognitive and motivational barriers to hypoglycaemia avoidance will help those with IAH and SH that has persisted after conventional structured education in flexible intensive insulin therapy to recover awareness and reduce $\mathrm{SH}$ experience. We therefore designed an intervention that uniquely includes a curriculum that addresses these cognitive and motivational barriers. The curriculum for the 6 -week intervention was codesigned by researchers, diabetes specialists, diabetes educators, people with diabetes and psychologists led by clinical psychologist NDZ. It was devised to be delivered by two diabetes educators, supervised by a clinical psychologist. A second curriculum was constructed for training the educators. A pilot study showed the intervention reduced $\mathrm{SH}$ rates from well above to below the reported average over the following year. ${ }^{41}$ Patients, family members and educators were interviewed. ${ }^{42}{ }^{43}$ Their comments were incorporated into a revised curriculum, the Hypoglycaemia Awareness Restoration Programme for People With Type 1 Diabetes and Problematic Hypoglycaemia Persisting Despite Optimised Self-care or HARPdoc. The trial reported here explores the clinical, cost and implementation effectiveness of HARPdoc.

Choice of comparator was complex, as the participants to be recruited into this trial will have been exposed to routinely available options, and they are a vulnerable patient group by virtue of their SH. BGAT, described below, was selected. ${ }^{27-29}$ The UK's National Institute for Health and Clinical Excellence (NICE) recommended BGAT for T1DM adults with problematic hypoglycaemia, ${ }^{44}$ although BGAT is not currently offered in the UK and has not formally been tested in people who have already completed structured education in flexible intensive insulin therapy as offered there that should have minimised SH risk. ${ }^{45}$ 
The main trial objective is therefore to compare the impact of the two programmes on hypoglycaemia experience, particularly SH experience, at 1 and 2 years after participation.

\section{METHODS AND ANALYSIS Trial design}

This is a randomised parallel two-arm trial of group therapies with rate of $\mathrm{SH}$ expressed per year measured at 1 and 2 years postrandomisation as two primary endpoints, comparing the novel psychotherapeutically enhanced education intervention HARPdoc with the control intervention BGAT. The trial was started in March 2017, and the estimated primary completion date is July 2021.

\section{Study setting}

The trial will be run in specialist care diabetes centres that support adults with T1DM: King's Health Partners (comprising King's College and Guy's and St Thomas' Hospitals NHS Foundation Trusts, London, UK); Sheffield Teaching Hospitals NHS Foundation Trust, UK; Royal Bournemouth Hospital, Bournemouth, UK, and the Joslin Diabetes Center, Boston, Massachusetts, USA. All provide structured education in flexible insulin therapy (DAFNE, BERTIE (Bournemouth Type 1 diabetes education) or the Joslin programme, DO-IT or Diabetes Outpatient Intensive Treatment), have clinical capability in use of CSII and CGM and routinely receive tertiary referrals for problematic hypoglycaemia.

Inclusion and exclusion criteria are listed in table $1 .^{24647}$

Participants who have expressed interest in the study, have consented and have IAH but do not meet all the trial inclusion criteria may be included in courses as 'Non-randomised participants' to ensure there are at least four people in each group, which is essential for creating the group format, allowing for one drop out. The information sheet for these participants is available in online supplementary file 1 .

\section{The interventions}

Hypoglycaemia Awareness Restoration Programme for People with Type 1 Diabetes and Problematic Hypoglycaemia Persisting Despite Optimised Self-care

The curriculum incorporates re-education in the mechanisms, outcomes, treatment and prevention of hypoglycaemia and hypoglycaemia unawareness. The novelty lies in incorporating a motivational interviewing (MI) approach to elicit and strengthen commitment to change and cognitive behaviour theory (CBT) to identify and restructure unhelpful cognitions. Three 'thinking traps' (based on qualitative research with people with $\mathrm{IAH}^{3741}$ ) will be introduced using cartoon metaphors and visual aids to make them more accessible. The first trap is the belief that one could 'soldier on' through hypoglycaemic episodes and delay treatment; the second that there were no important adverse outcomes to hypoglycaemia and/or IAH and the third overestimation of the risks of intermittent hyperglycaemia. Other psychological techniques used in regular education will be retained: social learning theory; a group structure; patient empowerment and an enabling, non-judgemental approach.

The curriculum will be delivered by two diabetes educators, to four to eight participants, over 6 weeks (table 2). In weeks 1-3, participants meet weekly in full-day sessions. They cover the pathophysiology, presentation, detection and treatment of hypoglycaemia and IAH and use MI to support behaviour change and encourage small changes, with CBT-informed strategies to address unhelpful cognitions. In weeks 4 and 5, participants will try out their new skills/strategies, with two individual support appointments (face to face or telephone). Week 6 is a final group session, focusing on relapse prevention and support for relatives who will be invited to participate, with their own session led by the psychologist. Group follow-up sessions (following an MI approach) will be scheduled at data collection times of 3, 6 and 12 months post-randomisation.

Scheduled supervision will be provided to HARPdoc educators by the study clinical psychologist, with debriefing sessions, weekly during the 6 week courses, and at 3, 6 and 12 month follow-ups, with further sessions available to support educators providing unscheduled one-to-one sessions to participants over the first year. In these, the educators will be able to describe the participants' progress and gain feedback on how to manage issues that arise.

\section{Blood Glucose Awareness Training}

BGAT is a well-established manualised intervention that is suitable for group or one-to-one delivery with a single diabetes educator. ${ }^{27}$ It teaches participants to focus on new ways of predicting extremes of blood glucose, through paying greater attention to internal cues (feelings and experiences) and providing greater understanding of external cues-drivers of high and low blood glucose. BGAT reviews the pharmacodynamics of insulin and glucose absorption from meals with careful and systematised recording and reflection on participants' own data. It is designed in eight classes, originally envisaged as each taken over 2 hours. In the present study, groups of participants will work through the BGAT manual text, led by one diabetes educator. For practical reasons (participants will be booked into courses before knowing which course they will receive), the BGAT curriculum will be delivered in four group sessions (two sessions in single-day attendances in weeks 1, 2, 3 and 6), with an optional telephone/Skype follow-up during weeks 4 or 5 . The curriculum will not be extended to ensure that group sessions necessarily occupy the same hours as HARPdoc.

\section{Training the educators}

A 3-day workshop was held during the planning of the study, which included protocol discussion, intervention 
Table 1 Inclusion and exclusion criteria

\begin{tabular}{ll}
\hline & Criterion \\
\hline Inclusion criteria & \\
Participants will & $\begin{array}{l}\text { be aged } \geq 18 \text { years. } \\
\text { have had T1DM for at least 4years. }\end{array}$
\end{tabular}

have been experiencing problematic hypoglycaemia for at least one yea

be willing to comply with the study design, including performance of glucose self-monitoring at least four times a day.

be able to communicate in written and spoken English and give written informed consent.

\section{Exclusion criteria}

\section{People with}

type 2 diabetes, or T1DM and intact

hypoglycaemia awareness.

no previous attendance at a

structured education programme.

lack of fluency in spoken English.

current pregnancy.

severe mental disorders

comorbidities other than diabetes

contributing to hypoglycaemia risk.
Impaired awareness of

hypoglycaemia

AND

having had $>1$ episode of $\mathrm{SH}$, with at least one since starting the current treatment modality and having had more than one episodes of $\mathrm{SH}$.
Explanation

References

defined clinically, having started insulin therapy within a year of diagnosis and/or having a history of diabetic ketoacidosis).

Gold and/or Clarke score of $\geq 4$.

episodes requiring assistance of another person to actively administer carbohydrates, glucagon or take other corrective action, because of impaired cognitive function and which may include episodes that were not treated by another but included loss of consciousness or seizure. training and creating appropriate course documents for UK and US centres. HARPdoc educator training was consolidated over 6 weeks following initial training, through weekly emailed homework practice and review by the study psychologist of recorded simulated one-to-one sessions with a medical actor to support educators reach the required skills competency. Care has and will be taken to keep the two groups of educators separate, to avoid contamination, throughout the study. such as DAFNE, BERTIE, DO-IT or the equivalent as judged by the investigator

Participants who continue to experience $\mathrm{SH}$ episodes 6 months after they have stopped breast feeding may be included.

schizophrenia, manic depression, depressive psychosis, active suicidal ideation, learning disability, dementia (either an existing diagnosis or a MiniMental State Examinations score of less than 24), alcohol and substance dependence, personality disorders); cognitive impairment independent of hypoglycaemia;

(eg, inadequately treated Addison's disease or growth hormone deficiency or hypothyroidism; untreated coeliac disease; uncontrolled gastroparesis; end-stage renal disease), which must have been checked since the onset of problematic hypoglycaemia.

a diagnosis of eating disorder. 
Table 2 HARPdoc: the curriculum

\begin{tabular}{ll}
\hline Format $\quad$ Topics \\
\hline Week 1: how low can you go?
\end{tabular}

Full-day group programme Introduction to the course and goal setting

Education topics What is a hypo?

Consequences of severe hypos.

Model of hypo unawareness.

Novel hypo awareness signs

Teaching the body scan.*

Complete individual severe hypo risk assessment.

Modify goal setting based on education.

Homework Estimating bg levels; doing a body scan and noting any novel hypo signs.

Week 2: the balancing act

Full-day group programme Education topics How to anticipate peaks/nadirs of insulin action.

$\mathrm{CHO}$ counting and matching.

Modifications for exercise/physical activity.

Behavioural plan

Homework Estimating bg levels (as above) and noting insulin/diet/activity (biological factors) and making insulin/food adjustments.

Week 3: thinking traps

Full-day group programme

Patients review answers to A2A questionnaire.

Education topics. Concept of thinking traps.

Pros/cons of each one.

How thinking traps interact with hypo management.

Group discussion about more helpful thoughts.

Strategies to support patients in their social context.

Thinking traps plan.

Homework Estimating bg levels (as above) and noting thoughts in response to bg level (psychological factors).

Weeks 4 and 5

One hour each week individual appointments for face-to-face, telephone or internetbased contact with educator.

Reviewing patient progress/plans and provide troubleshooting support.

Week 6: keeping on track

Full-day group session Revise core education topics with group.

Invitation to bring significant other and Q\&A session.

Group discussion on progress made.

Group discussion on future challenges/relapse prevention.

Opportunity for final trouble shooting.

Individual relapse prevention plans.

*Teaching patients to scan their body for any subtle cues that may indicate being low.

A2A, Attitudes to Awareness (of hypoglycaemia); bg, blood glucose; CHO, carbohydrate; HARPdoc, Hypoglycaemia Awareness Restoration

Programme for People with Type 1 Diabetes and Problematic Hypoglycaemia Persisting Despite Optimised Self-care.

any contacts made to support course learning outside the main course delivery.

\section{Patient and public involvement}

Improvement in awareness and prevention of hypoglycaemia was identified explicitly as a research priority (number 6 of 10) by people with type 1 diabetes and their carers collaboratively with researchers and healthcare professionals in a James Lind Alliance exercise; avoidance of hypoglycaemia was a target of all the other nine. ${ }^{48}$
Reducing hypoglycaemia burden also featured in the public consultation of priorities for revising the guidelines for the management of adults with type 1 diabetes for the UK's NICE. ${ }^{44}$ People with diabetes were involved in creating the original curriculum for the pilot, and people with diabetes and carers were formally involved in the preparation of the revision. The trial has its own user group, members of which reviewed the protocol, advised on the potential burden of participation and informed 
the choice of primary endpoint and the name of the new intervention. The group continue to advise and actively assist recruitment, participating in the Trial Management Group (TMG).

\section{Outcomes}

Our primary outcome is the difference in rate of SH events (number of events over preceding year) between the two arms, measured using 12-month and 24-month anonymised recall forms and adjusted for baseline SH rate. Significantly lower event rates for HARPdoc as compared with BGAT at either 12 months postrandomisation, 24 months postrandomisation or both will be considered to indicate superiority of the HARPdoc treatment.

\section{Secondary outcomes}

We will also compare between the two groups parameters to assess aspects of hypoglycaemia experience and other impacts of the intervention adjusted for baseline and other previous timepoints where appropriate at 12 and 24 months postrandomisation (table 3 , refs ${ }^{46} 4749-53$ ).

As change in therapy modality such as new uptake of pumps and/or sensors (CGM) will impact on SH rate, we will describe the numbers of people making such changes in each group, namely: introduction of novel (to that participant) pump, CGM, automated insulin infusion adjustment technology, use of retrospective intermittently monitored 'Flash' glucose monitoring, islet or pancreas transplantation and psychiatric or psychological therapy external to the trial.

\section{Tertiary objectives}

Outside the main study analyses, we also propose to compare between groups at 12 and 24 months, adjusting for baseline where appropriate:

1. Exposure to plasma glucose readings of: (1) under $3.9 \mathrm{mmol} / \mathrm{L}$ and (2) under $3 \mathrm{mmol} / \mathrm{L}$ as event rates and percent of all readings made per patient per week on home glucose monitoring records, including retrospective intermittently monitored 'Flash' CGM glucose readings at time of monitoring from 2 weeks (14 days) prior to data collection.

2. Total score on Hypoglycaemia Cues Questionnaire. ${ }^{54}$

3. Total Score on a diabetes-specific Quality of Life Questionnaire $^{55}$ and Multidimensional Scale of Perceived Social Support ${ }^{56}$ to assess quality of life and social support.

\section{Health economics analysis}

An economic evaluation will examine the cost-effectiveness of HARPdoc compared with BGAT within a cost-per quality adjusted life year (cost-utility) framework, ${ }^{57}$ using trial data from all UK sites. The base-case economic evaluation will be carried out from and NHS/personal social services perspective. As part of a sensitivity analysis around base-case findings, a secondary 'societal' analysis will assess whether inclusion of employment-related costs linked to severe hypo events (specifically work time lost) and the costs of service contacts outside of the NHS (specifically psychological treatment services) impacts on base-case cost-effectiveness conclusions. ${ }^{58}$

\section{Parallel implementation study}

Under a separate protocol, researchers, participants and people who did not participate will be invited to contribute to an implementation study, which will aim formally to evaluate the way in which each trial intervention is being delivered in the context of the local services across study sites. This mixed methods study will collect a range of implementation outcome metrics (intervention acceptability, appropriateness and feasibility; intended adoption) from intervention providers and users; study the clinical and service user context in which the interventions are being delivered; and finally study the link between the quality of delivery of the trialled interventions and the expected improvements in the study endpoints. ${ }^{59}$ In carrying out a parallel evaluation of clinical and implementation endpoints and in correlating the two sets of endpoints as part of the overall trial, we are conducting what has recently been termed a 'hybrid effectiveness-implementation type II' study. ${ }^{60}$ The implementation study has a study protocol, coauthored with service users and reported separately in detail. ${ }^{61}$

\section{Planned ancillary analyses}

These are listed in table 4 (including refs ${ }^{5062}$ ).

\section{Participant timeline}

The timeline for the study is shown in table 5. Potential participants will be invited to express interest in the study by their diabetes healthcare professional. Those expressing interest will receive the participant information sheet (online supplementary file 2) and an opportunity to discuss the study with a member of the research team. If they wish to proceed, they will sign a consent form and be allocated a unique study number. Initial screening will include recall of SH episodes over the previous 1 and 2 years and of hypoglycaemia awareness status (figure 1A). Participants will start prospective recording of $\mathrm{SH}$, using study forms (figure 2). Baseline data collection may be contemporaneous with screening if the course is due in less than 1 month. Participants who have undergone significant change in therapy since screening and have had resolution of their hypoglycaemia issues will not be randomised and will be replaced. Follow-up sessions will be at 3, 6, 12, 18 and 24 months after randomisation and include 2-hour group revision sessions for HARPdoc participants in the first year.

\section{Sample size}

The study is powered as a superiority study. We estimate that 96 participants will give us $90 \%$ power to detect a difference in our primary endpoints at $2.5 \%$ level of significance with a $20 \%$ attrition rate. To ensure adequate recruitment to each course, we may recruit to a maximum of 120 .

The sample size calculation used the published data on the base rates and impact of the two interventions 


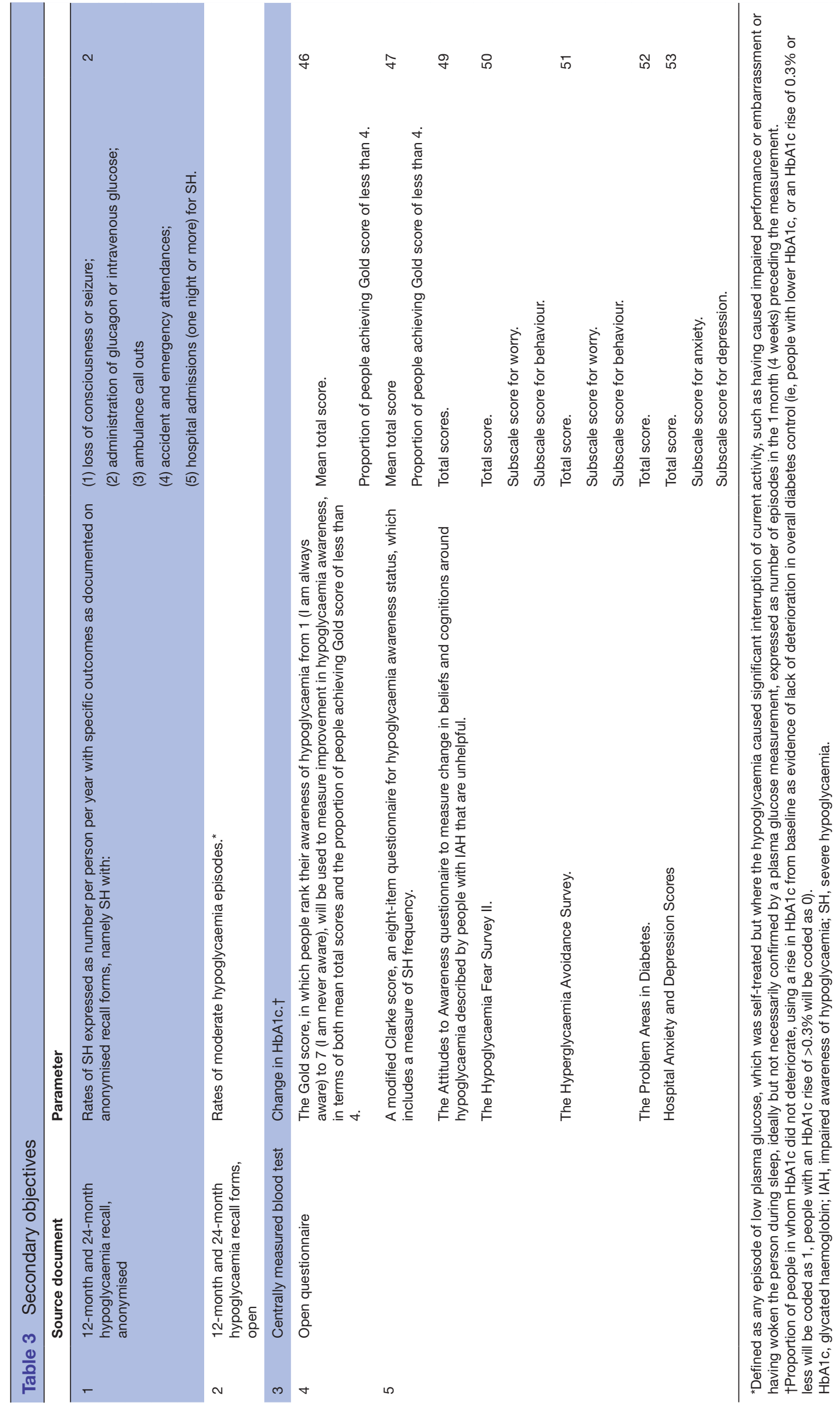


Table 4 Ancillary analyses

\begin{tabular}{llll}
\hline Study title & Population studied & Outcomes & References \\
\hline 1 & Outcomes for people with low & Participants expressing low & Primary outcome and secondary \\
concern about hypoglycaemia & concern on the baseline & outcomes 1, 2 and 4. \\
at baseline. & Hypoglycaemia Fear Survey & \\
& II, defined as those with & \\
& scores s25th percentile in a & \\
& routine clinic survey. &
\end{tabular}

2 Comparison of different methods for collecting data about $\mathrm{SH}$ in studies.
All participants.

Descriptive study comparing rates of $\mathrm{SH}$

recalled on anonymised 12-month and

24-month recall forms (figure 1) with data collected on:

1. Open recall forms.

2. Relatives' recall forms.

3. GP recall forms.

4. Items 3 and 4 of the Clarke scores.

5. Immediate reporting on form $A$ (adjudicated).

6. Monthly reports on form B.

\begin{tabular}{|c|c|c|c|}
\hline 3 & $\begin{array}{l}\text { Descriptive analysis of the } \\
\text { fidelity with which each } \\
\text { intervention is delivered. }\end{array}$ & $\begin{array}{l}\text { Audio tapes of group sessions } \\
\text { made with participants' } \\
\text { consent (relatives' information } \\
\text { sheet, online supplementary } \\
\text { file 3). }\end{array}$ & $\begin{array}{l}\text { Trial psychologist and independent } \\
\text { assessors will use a novel rating tool } \\
\text { adapted from the Assessment of } \\
\text { Motivational Interviewing Groups - } \\
\text { Observer Scale for quality assurance and } \\
\text { evidence of cross contamination. }\end{array}$ \\
\hline 4 & $\begin{array}{l}\text { Hypoglycaemia recorded by } \\
\text { CGM }\end{array}$ & $\begin{array}{l}\text { Substudy of } 24 \text { ( } 12 \text { in each } \\
\text { group) participants who will } \\
\text { undertake blind CGM for } \\
2 \text { weeks at baseline and at } \\
12 \text { months postrandomisation. }\end{array}$ & $\begin{array}{l}\text { Differences in exposure to CGM measures } \\
\text { of hypoglycaemia, namely, (1) alerts } \\
\text { (number per week); and (2) glucose } \\
\text { recordings of: }(\mathrm{A}) \text { under } 3.9 \mathrm{mmol} / \mathrm{L} \text {; } \\
\text { (B) under } 3 \mathrm{mmol} / \mathrm{L} \text {; and }(\mathrm{C}) \text { under } \\
2.2 \mathrm{mmol} / \mathrm{L} \text {, as both event rate (number } \\
\text { of events of at least } 15 \text { min duration per } \\
\text { person per week, separated by at least } \\
15 \text { min from an earlier event) and duration } \\
\text { (total time spent at these values in hours } \\
\text { and minutes per week). }\end{array}$ \\
\hline
\end{tabular}

CGM, continuous glucose monitoring; GP, general practitioner; $\mathrm{SH}$, severe hypoglycaemia.

in this highly selected population. The mean rate of $\mathrm{SH}$ at the start and end of the pilot for HARPdoc, DAFNEHART, was 19 per year falling to 0.5 per subject year at 12 months. ${ }^{41}$ For the present study, we considered also a more conservative estimate of HARPdoc success, namely two SH events per person per year. For BGAT, we used an outcome of 3.8 episodes per year, the mean achieved value of three published studies. ${ }^{27-29}$ We inflated the sample size to take account of within-group correlation and adjusted for therapist group, for which the intraclass correlation has been estimated as $0.02 .{ }^{63}$ It is envisaged that therapy groups will have $6-8$ patients giving a design effect of $1+0.02(8-1)=1.14$. We further adjusted for multiple comparison with the use of Bonferroni correction in two endpoints (12 and 24 months) (corrected alpha $=0.025 \%)$.

\section{Assignment of interventions}

Once a centre has recruited 8-16 eligible participants, their data will be checked for eligibility by the clinical trial manager and entered into the randomisation system, provided by the King's Clinical Trials Unit, no more than a week before the course start date.

The randomisation system randomly sequences the order of the participants and enters them into the study stratifying by country and by technology use (use of pumps and/or CGM systems, not including use of intermittently monitored retrospective CGM ('Flash') glucose monitoring). Non-randomised participants will undertake the same procedures, but their data will not be included in the main trial analysis.

Should anyone become ineligible between screening and randomisation (eg, if they have changed their treatment modality and ceased to have problematic hypoglycaemia), or they wish to withdraw, they can be replaced by a newly recruited person. Once a person is randomised to the study, they remain in the study unless he or she chooses to withdraw or they are withdrawn. 


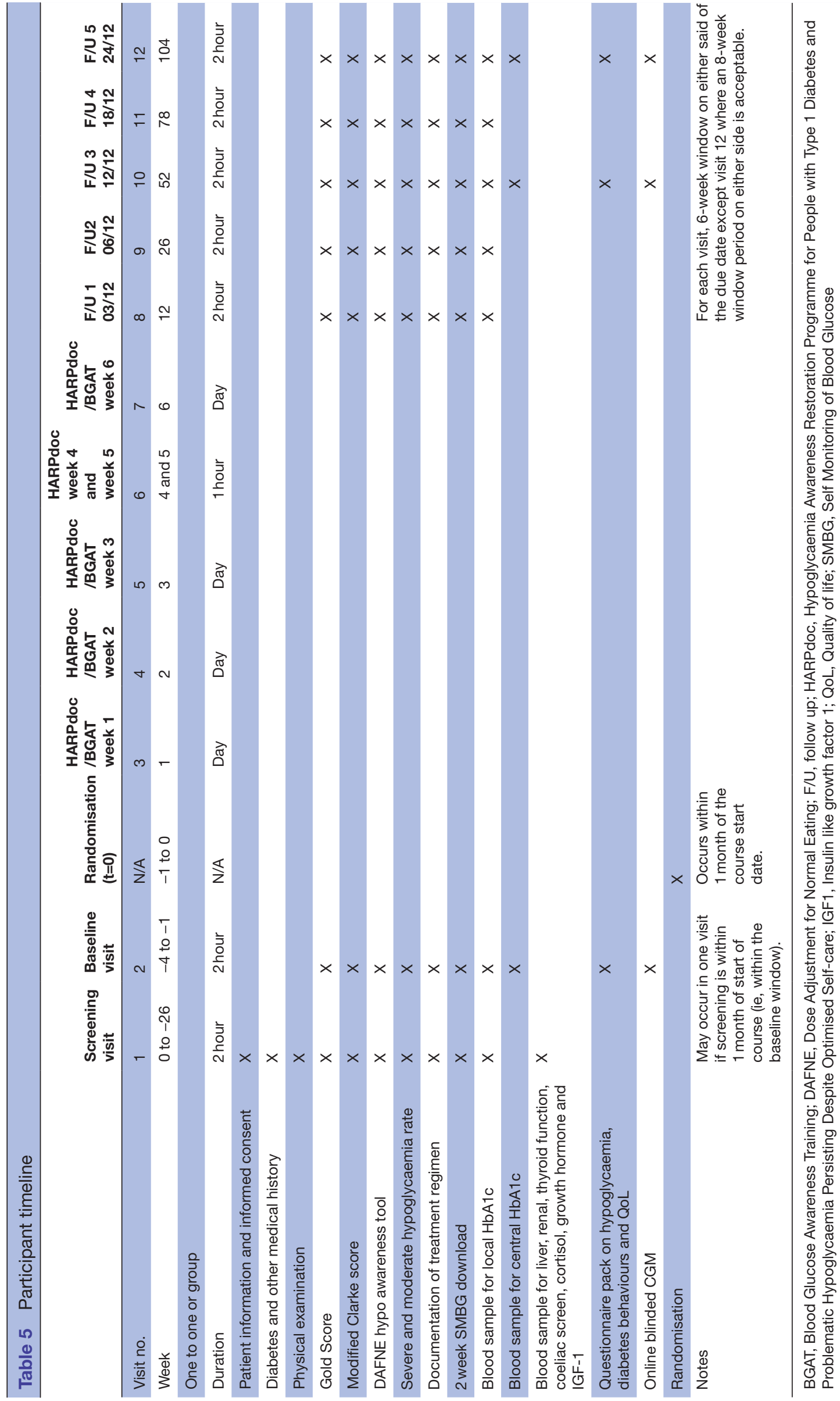

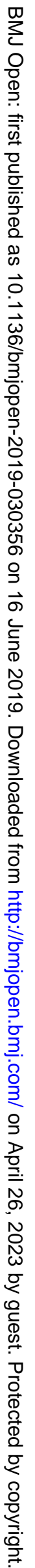



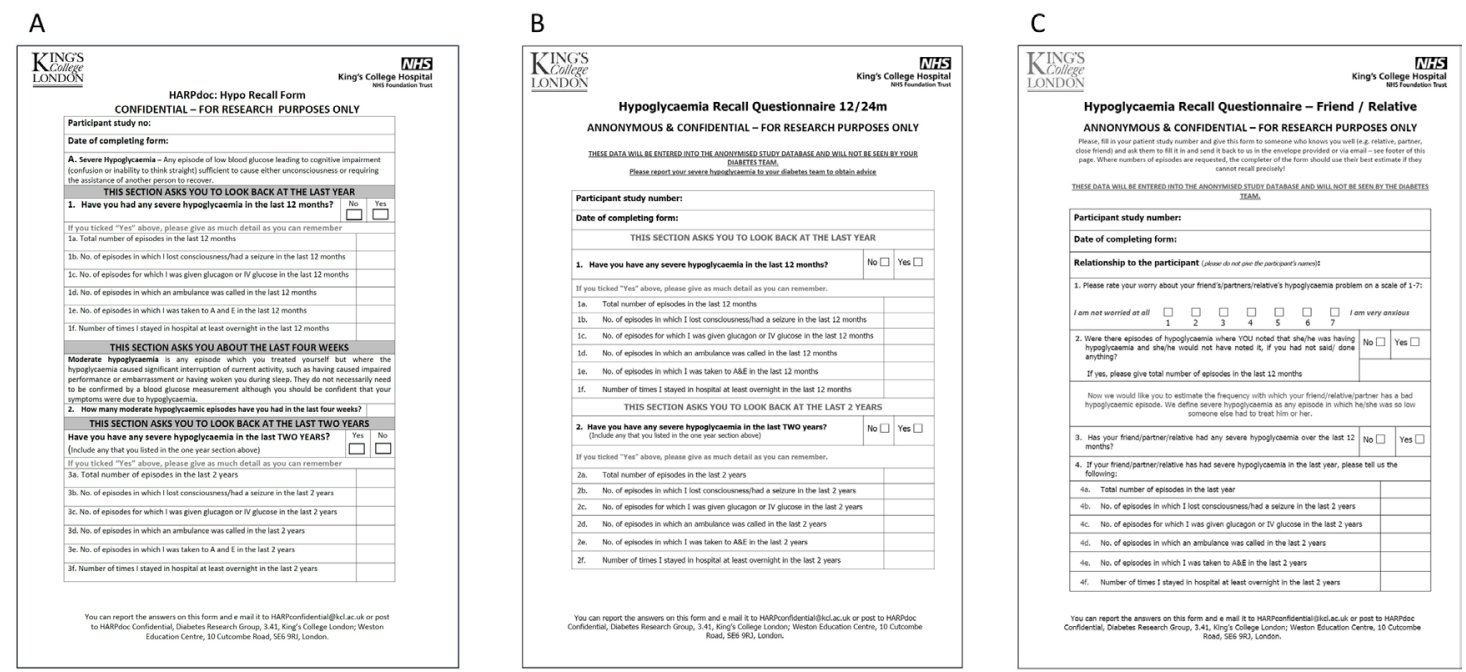

Figure 1 Twelve-month and 24-month hypoglycaemia recall forms: (A) open, (B) anonymised and (C) relatives.

The clinical trial manager will be informed of the randomisation outcome and relays the information to the centre, usually all by e mail. Local educators inform participants which group they will attend by telephone, email or in person on the morning of the first day, where practical. Where possible, HARPdoc and BGAT courses will be run simultaneously.

\section{Blinding}

Participants researchers and participants' usual care providers cannot be blinded to course allocation once the randomisation is complete. The trial statisticians remain blinded to group allocation throughout.

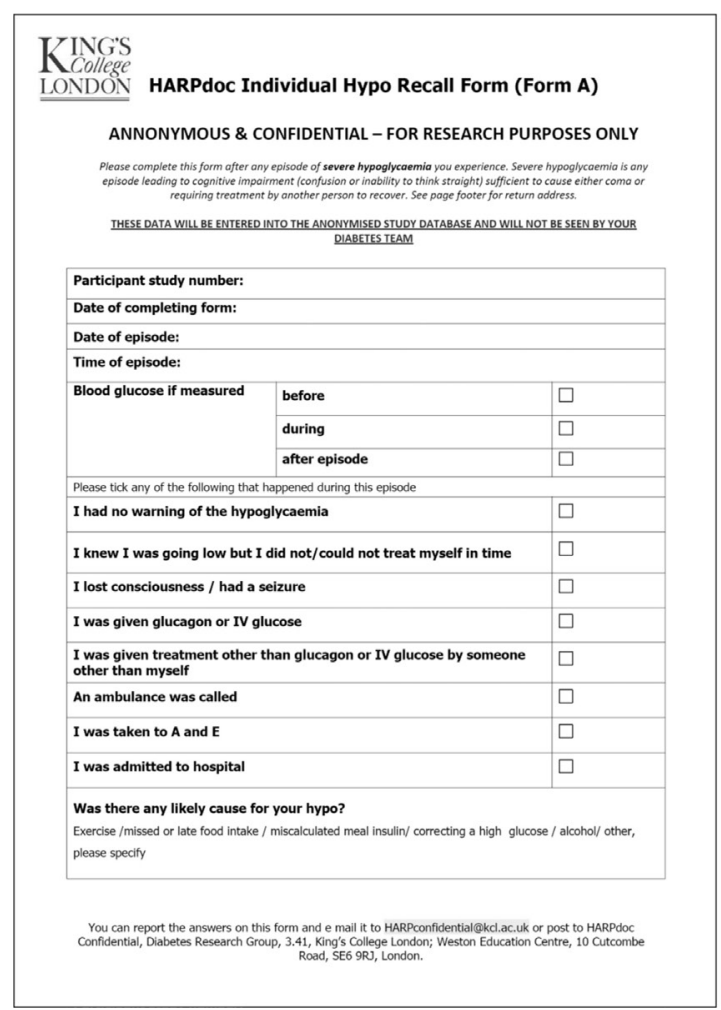

\section{Data collection}

Apart from a blood test for glycated haemoglobin, taken at baseline, 12 and 24 months and sent to Viapath at King's College Hospital London for central measurement by High Pressure Liquid Chromatography (HPLC) methods, all outcome data will be collected by questionnaires. Validated questionnaires are listed in table 3 and above. The questionnaires for collecting data on hypoglycaemia experience are purpose-built for the study, based on tools used in earlier research, particularly the UK Hypoglycaemia Study Group data forms. ${ }^{64}$ Participants will complete two versions of forms detailing their recall

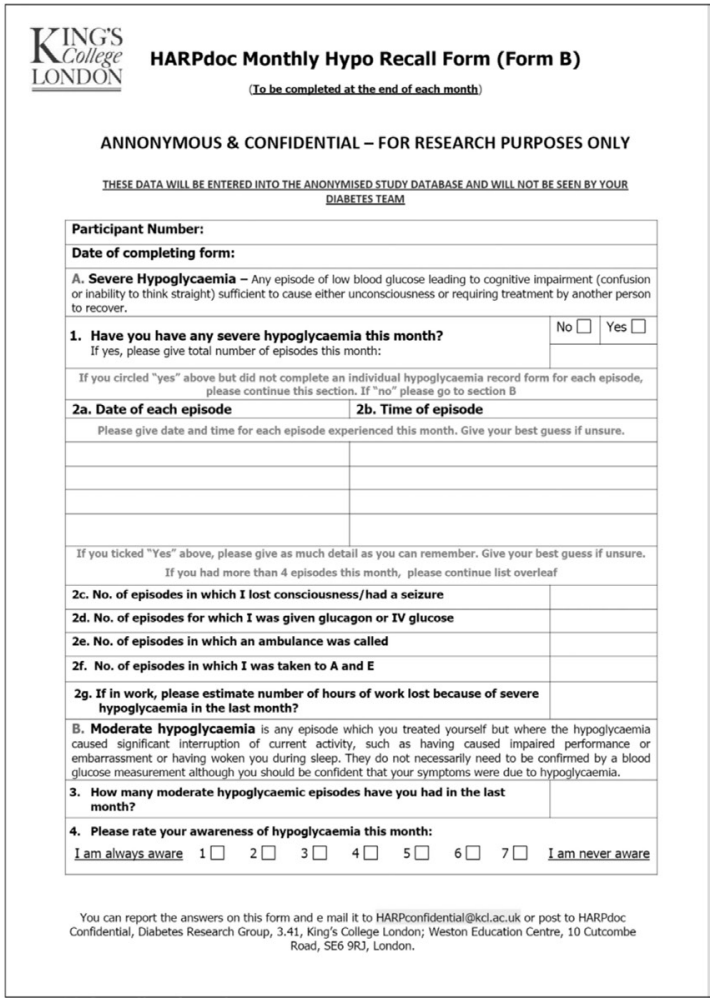

Figure 2 Forms $A$ and B for immediate and monthly reporting of severe hypoglycaemia. 
of SH. One (Figure 1A) is open and can be discussed with the researchers to be confident of shared definitions of $\mathrm{SH}$. The other (Figure 1B), which will be used for the primary outcome, is anonymised, mailed to a non-clinical study data manager for direct entry into the study electronic database, without clinical review. This is because of evidence of under-reporting of SH since the introduction of European guidelines mandating loss of driving privileges for people reporting more than one $\mathrm{SH}$ in a year. ${ }^{65}$ A further questionnaire is included for the participant to give to a close relative or friend, for an independent assessment of their $\mathrm{SH}$ experience (figure 1C). Data on SH involving unscheduled care will be collected from primary care services.

Forms for anonymously recording $\mathrm{SH}$ immediately (form A) and monthly (form B) will be mailed out by local centres, and their research teams remind participants to return the monthly forms (figure 2). All anonymised forms will remind participants that their diabetes team will not see these data and to contact their educators if they want advice.

The open 12-month and 24-month recall form, Gold, DAFNE and Clarke scores for hypoglycaemia awareness and all the other questionnaires will be sent to participants in a booklet, with the anonymised and the relatives' $\mathrm{SH}$ recall forms in the week before courses and before 12-month and 24-month follow-up.

Should a participant decide to withdraw, efforts will be made to report the reason for withdrawal. If the participant has been randomised, efforts will be made to continue to obtain follow-up data. Participants will remain under services that routinely collect the data of our main endpoints (SH rate, HbAlc and Gold score). We will collect case record data \pm 1.5 months of due dates via the participants' usual diabetes care provider, subject to participant permission.

\section{Data management}

Data will be collected onto paper clinical record forms (CRFs), then uploaded by research administrative staff into a study-specific electronic database constructed and managed for the trial by the King's College London (KCL) Clinical Trials Unit using the participant's unique study identifier. The clinical trial manager and the study data manager will review the electronic data base and will visit each centre to check the data entry against the paper CRFs. Patient data will be managed in line with the General Data Protection Act 2018. The trial will be monitored by the King's Clinical Trial Office. The chief investigator will act as custodian for the trial data.

\section{Statistical methods}

The main statistical analyses will be carried out by the trial statistician who will be blind to the group randomisation until the main analyses are complete. The analyses outlined in this strategy will be primarily based on intention to treat. A per-protocol analysis will also be conducted in which the primary outcome will also be compared between groups, removing data of participants who:

1. Do not complete their intervention as defined above.

2. Delay undertaking their intervention by more than 2 months after randomisation.

3. Are later found to have an exclusion criterion that was missed, including those who become pregnant.

4. Undertake islet or pancreas transplant.

Data from participants who become pregnant or undergo transplantation will be retained until the event. The numbers of participants who escalate their therapy to include new technology will be documented for each group.

The first stage of analyses will be a descriptive model of the data to assess completeness of data. Participant-level baseline variables will be described both overall and by randomised groups, country and centre. Patterns of missing data will be described.

The primary outcome will be analysed using Poisson or negative binominal linear mixed models, depending on the dispersion of the data, to model the difference in $\mathrm{SH}$ rate (number of $\mathrm{SH}$ events over the preceding year) at 1 and 2 years' postrandomisation. The Poisson or negative binomial linear mixed models will be adjusted for baseline SH rate (events in the year preceding baseline) and stratification factors (country and using technology). The model will use a random intercept for each therapy group to account for clustering. A three-level hierarchical model will be employed with all time points included as repeated measures in the model (12 and 24 months) to improve power and take into account clustering of the observation at patient and group therapy level.

These models use maximum likelihood estimation and thus allow for missing outcome data under the missing at random (MAR) assumption. Associations between postrandomisation variables and missingness will be dealt with by multiple imputation, again under the MAR assumption. Departures from this assumption will be assessed with a sensitivity analysis.

Secondary outcomes will be assessed with a similar methodology for the primary outcomes, using generalised linear mixed models depending on the type of outcome (normal, binary and count). The questionnaires to be used have validated methods of scoring, and the scores will be analysed as described.

\section{Monitoring}

A Data Monitoring Committee (DMC), Trial Steering Committee (TSC) and TMG comprising all PIs, the statisticians and chairman of the trial's user group have been established to oversee the study. The DMC and TSC are described below. The DMC will review $\mathrm{SH}$ rates and can stop the trial if one arm is dangerously underperforming. The Chief Investigator (CI) and/or clinical trial manager will visit each site at setup and at least annually during the study. The trial will be monitored by the King's Health Partners Clinical Trials Office to ensure compliance with Good Clinical Practice. 
Adverse events will be recorded in the medical records. Data on diabetic ketoacidosis, falls and fractures and starting or stopping therapy (medical or psychotherapeutic) for psychiatric events will be recorded in the CRFs from time of consent to final data collection. SH will be collected throughout the trial using trial documentation (12 and 24month recall and forms A and B) and not reported as an adverse event.

\section{Ethics}

At worst, participants will remain at their baseline $\mathrm{SH}$ risk. $\mathrm{SH}$ rates will be monitored by the DMC. For individual participants, if their SH persists unchanged, other strategies will be explored. As participants should have already experienced optimal conventional care, these options are limited but may include offers of technology for glucose sensing or insulin delivery (CGM and pump) that were not previously available to that participant and referral for islet or pancreas transplantation. Requirement for other therapies, such as psychological interventions, will be assessed on an individual basis. It should however be noted that desire to engage with a new technology or treatment that has previously not been successful or acceptable to a participant may well be a positive result of engaging in a study course and previous exposure to such technologies will be collected at baseline.

\section{DISSEMINATION}

We will report the results of the trial to the participants through written and verbal reports, with further dissemination at national/international conferences and in peer-reviewed scientific journals. We will also report to people with diabetes and their carers through publications (electronic and/or paper) of the Juvenile Diabetes Research Foundation and the South London National Institute of Health Research Collaboration for Leadership in Applied Health Research and Care South London and by presentations to patient groups. There has been interest from the US healthcare provider, Kaiser Permanente, with which we hope to work on scaling up the successful intervention in the USA.

\section{Author affiliations}

${ }^{1}$ Diabetes Department, Denmark Hill Campus, King's College London Faculty of Life Sciences and Medicine, London, UK

${ }^{2}$ Department of Diabetes, King's College Hospital NHS Foundation Trust, London, UK ${ }^{3}$ Department of Psychiatry and Behavioral Sciences, University of Virginia, Charlottesville, Virginia, USA

${ }^{4}$ School of Medicine and Biomedical Sciences, University of Sheffield Faculty of Medicine Dentistry and Health, Sheffield, UK

${ }^{5}$ Bournemouth Diabetes and Endocrine Centre, Royal Bournemouth Hospital, Bournemouth, UK

${ }^{6}$ Joslin Diabetes Center, Harvard University, Cambridge, Massachusetts, USA

${ }^{7}$ Department of Diabetes, Guy's and Saint Thomas' Hospitals NHS Trust, London, UK ${ }^{8}$ Department of Biostatistics, Department of Health Services and Population Research, Institute of Psychiatry Psychology and Neuroscience, London, UK ${ }^{9}$ Centre for Implementation Science, Health Sciences and Population Research Department, Institute of Psychiatry Psychology and Neuroscience, London, UK
Acknowledgements The authors would like to thank the patients who are taking part in this study. The research is sponsored by King's College Hospital National Health Service (NHS) Foundation Trust and King's College London. The clinical sites taking part are Guy's and St Thomas' NHS Foundation Trust, the Joslin Diabetes Center, Boston, Massachusetts, USA, King's College Hospital NHS Foundation Trust and the Royal Bournemouth General Hospital, Bournemouth, Dorset, UK, with Patient Identification Sites at St Mary's Hospital, Paddington (Professor Nick Oliver), East Kent Hospitals University NHS Foundation Trust (Dr Stonny Joseph), Royal Sussex County Hospital, Brighton and Sussex Universities Hospitals (Dr Ali Chakera), Royal Free London NHS Foundation Trust (Dr Miranda Rosenthal) and St George's Hospitals NHS Foundation Trust (Drs Nicola Neary and Arshia Panaloo). The authors would also like to thank Caroline Murphy and the KCL Clinical Trial Unit for providing the randomisation procedures and the electronic data base; Marilyn Ritholz for additional psychological support; Sean Haywood and Karolina Burakowska for central trial administration; diabetes educators Monet Bland, Carly Devin, Victoria Francis, Val Gordon, Emma Jenkins, Lois Maurer, Sarah Newall, Nicole Patience, Jacqueline Ryder, Jill Rimmel, Helen Rogers and Melanie Weiss, and researchers Astrid Atakov-Castillo, Sharon Caunt, Tanith Changuion, Linda Greaves, Gail Hann, Sue Hudson, Chloe Nisbet, Marius Racz, Elsa Redfern and Navone Thompson, and our patient representative group Mike Kendall (chair), Richard Lane, OBE, Lis Warren, Mel Stephenson, Vicky Ruzala and Arthur Durrant.

Contributors SA wrote the first draft of the manuscript and designed this study, with input from all the listed authors. NDZ, with SA, HR, PC and SH, designed the DAFNE-HART/HARPdoc (Dose Adjustment for Normal Eating-Hypoglycaemia Awareness Restoration Training) programme and training curriculum for HARPdoc educators, with support from the UK DAFNE collaborative and patient group; ELS led this group on the update for the trial; LG-F wrote the original Blood Glucose Awareness Training (BGAT) programme and led the revision, with SA, MS, PJ and MK and support from the BGAT educators. MS and PC designed the continuous glucose monitoring substudy. KG, IB and LP wrote the statistical analysis plan and power calculations and commented on the final form of the protocol and choice of end points. AH designed the health economic study; MK supported by the Patient and Public Involvement (PPI) group and reviewed the protocol and all the patientfacing papers; DK, ET, SH and AB reviewed the protocol, supported the training of the educators and set up the study sites; $M Q$ runs the database and administers the trial. NS designed and leads the implementation science protocol. All authors have read and contributed significantly to the manuscript and approved its final form.

Funding This project is funded by the Juvenile Diabetes Research Foundation Project Grant no 4- SRA-2017-266-M-N, with additional support from the UK's National Institute of Health Research (NIHR) through the South London Collaboration for Leadership in Applied Health Research and Care South London (CLAHRC) at King's College Hospital NHS Foundation Trust, London, UK. NS, AH and IB are also members of King's Improvement Science, part of the NIHR CLAHRC South London based at King's College London and funded by King's Health Partners (Guy's and St Thomas' NHS Foundation Trust, King's College Hospital NHS Foundation Trust, King's College London and South London and Maudsley NHS Foundation Trust), Guy's and St Thomas' Charity, the Maudsley Charity and the Health Foundation. The study is sponsored jointly by King's College London (Professor Rezavi, susan. dickson@kcl.ac.uk) and King's College Hospital NHS Foundation Trust, (Research \& Innovation Office, kch-tr.research@nhs.net).

Disclaimer The funders had no role in the design, conduct or analysis of this study. The views expressed are those of the authors and not necessarily those of the Juvenile Diabetes Research Foundation International (JDRF), the NHS, the NIHR or the Department of Health and Social Care.

Competing interests SA is a member of the International Hypoglycaemia Study Group and has served on advisory panels for Roche, Medtronic and NovoNordisk. $A B$ has received honoraria from Astra Zeneca and Sanofi for speaking at educational events and sponsorship from Lilly and Janssen to attend conferences. SH has served as a consultant or speaker from Lilly, Novo Nordisk, Takeda, Boeringher Ingelheim, Mannkind, Sanofi, Zealand Pharma and UN-EEG. NS is the Director of London Safety and Training Solutions Ltd, which provides quality and safety training and advisory services on a consultancy basis to healthcare organisation globally.

Patient consent for publication Not required.

Ethics approval The protocol for the study has been approved by the London Dulwich Research Ethics Committee (IRAS number 216381), and the Health Research Authority with additional approvals for the US centre obtained from the Institutional Review Board of the Joslin Diabetes Center. 
Provenance and peer review Not commissioned; peer reviewed for ethical and funding approval prior to submission.

Author note The trial steering committee members are: Professor James Shaw, Newcastle University (chair), Richard Holt (chair) and Irene Stratton, Gloucester Hospitals NHS Foundation Trust; the independent data monitoring and ethics committee members are Amanda Adler (Addenbrookes Hospital Cambridge), Steve Sharp (MRC Epidemiology Unit, University of Cambridge) and Mark Evans (University of Cambridge).

Open access This is an open access article distributed in accordance with the Creative Commons Attribution Non Commercial (CC BY-NC 4.0) license, which permits others to distribute, remix, adapt, build upon this work non-commercially, and license their derivative works on different terms, provided the original work is properly cited, appropriate credit is given, any changes made indicated, and the use is non-commercial. See: http://creativecommons.org/licenses/by-nc/4.0/.

\section{REFERENCES}

1. Wild D, von Maltzahn R, Brohan E, et al. A critical review of the literature on fear of hypoglycemia in diabetes: Implications for diabetes management and patient education. Patient Educ Couns 2007;68:10-15.

2. International Hypoglycaemia Study Group. Glucose Concentrations of Less Than $3.0 \mathrm{mmol} / \mathrm{L}(54 \mathrm{mg} / \mathrm{dL})$ should be reported in clinical trials: a joint position statement of the american diabetes association and the european association for the study of diabetes. Diabetes Care 2017;4060:1553-76.

3. Lawton J, Rankin D, Elliott J, et al. Experiences, views, and support needs of family members of people with hypoglycemia unawareness: interview study. Diabetes Care 2014;37:109-15.

4. Dømgaard M, Bagger M, Rhee NA, et al. Individual and societal consequences of hypoglycemia: A cross-sectional survey. Postgrad Med 2015;127:438-45.

5. Gill GV, Woodward A, Casson IF, et al. Cardiac arrhythmia and nocturnal hypoglycaemia in type 1 diabetes--the 'dead in bed' syndrome revisited. Diabetologia 2009;52:42-5.

6. Pedersen-Bjergaard U, Pramming S, Heller SR, et al. Severe hypoglycaemia in 1076 adult patients with type 1 diabetes: influence of risk markers and selection. Diabetes Metab Res Rev 2004;20:479-86.

7. Patterson CC, Dahlquist G, Harjutsalo V, et al. Early mortality in EURODIAB population-based cohorts of type 1 diabetes diagnosed in childhood since 1989. Diabetologia 2007;50:2439-42.

8. Feltbower RG, Bodansky HJ, Patterson CC, et al. Acute complications and drug misuse are important causes of death for children and young adults with type 1 diabetes: results from the Yorkshire Register of diabetes in children and young adults. Diabetes Care 2008;31:922-6.

9. Skrivarhaug T, Bangstad HJ, Stene LC, et al. Long-term mortality in a nationwide cohort of childhood-onset type 1 diabetic patients in Norway. Diabetologia 2006;49:298-305.

10. Huxley RR, Peters SA, Mishra GD, et al. Risk of all-cause mortality and vascular events in women versus men with type 1 diabetes: a systematic review and meta-analysis. Lancet Diabetes Endocrinol 2015;3:198-206.

11. The Healthcare Quality Improvement Partnership NDA. National diabetes audit 2012-2013 report 2: complications and mortality, 2015.

12. Nathan DM, DCCT/EDIC Research Group. The diabetes control and complications trial/epidemiology of diabetes interventions and complications study at 30 years: overview. Diabetes Care 2014;37:9-16.

13. Mitrakou A, Ryan C, Veneman T, et al. Hierarchy of glycemic thresholds for counterregulatory hormone secretion, symptoms, and cerebral dysfunction. Am J Physiol 1991;260(1 Pt 1):E67-74.

14. Bolli $\mathrm{G}$, de Feo P, Compagnucci $\mathrm{P}$, et al. Abnormal glucose counterregulation in insulin-dependent diabetes mellitus. Interaction of anti-insulin antibodies and impaired glucagon and epinephrine secretion. Diabetes 1983;32:134-41.

15. Geddes J, Schopman JE, Zammitt NN, et al. Prevalence of impaired awareness of hypoglycaemia in adults with Type 1 diabetes. Diabet Med 2008;25:501-4.

16. Hopkins D, Lawrence I, Mansell P, et al. Improved biomedical and psychological outcomes 1 year after structured education in flexible insulin therapy for people with type 1 diabetes: the U.K. DAFNE experience. Diabetes Care 2012;35:1638-42.

17. Burki TK. Diabetes and driving. Lancet Diabetes Endocrinol 2013;1:e7-8.
18. Jacobson AM, Braffett BH, Cleary PA, et al. The long-term effects of type 1 diabetes treatment and complications on health-related quality of life: a 23-year follow-up of the Diabetes Control and Complications/Epidemiology of Diabetes Interventions and Complications cohort. Diabetes Care 2013;36:3131-8.

19. Amiel SA, Sherwin RS, Simonson DC, et al. Effect of intensive insulin therapy on glycemic thresholds for counterregulatory hormone release. Diabetes 1988;37:901-7.

20. Heller SR, Cryer PE. Reduced neuroendocrine and symptomatic responses to subsequent hypoglycemia after 1 episode of hypoglycemia in nondiabetic humans. Diabetes 1991;40:223-6.

21. George E, Harris N, Bedford C, et al. Prolonged but partial impairment of the hypoglycaemic physiological response following short-term hypoglycaemia in normal subjects. Diabetologia 1995;38:1183-90.

22. Cranston I, Lomas J, Maran A, et al. Restoration of hypoglycaemia awareness in patients with long-duration insulin-dependent diabetes. Lancet 1994;344:283-7.

23. Dagogo-Jack S, Rattarasarn C, Cryer PE. Reversal of hypoglycemia unawareness, but not defective glucose counterregulation, in IDDM. Diabetes 1994;43:1426-34.

24. Bolli GB, Pampanelli S, Porcellati F. Fanelli CG: Recovery and prevention of hypoglycaemia unawareness in type 1 diabetes mellitus. Diabetes. nutrition \& metabolism 2002;15:402-9.

25. Yeoh E, Choudhary P, Nwokolo M, et al. Interventions that restore awareness of hypoglycemia in adults with type 1 diabetes: a systematic review and meta-analysis. Diabetes Care 2015;38:1592-609.

26. Little SA, Leelarathna L, Walkinshaw E, et al. Recovery of hypoglycemia awareness in long-standing type 1 diabetes: a multicenter $2 \times 2$ factorial randomized controlled trial comparing insulin pump with multiple daily injections and continuous with conventional glucose self-monitoring (HypoCOMPaSS). Diabetes Care 2014;37:2114-22.

27. Cox DJ, Gonder-Frederick L, Polonsky W, et al. Blood glucose awareness training (BGAT-2): long-term benefits. Diabetes Care 2001;24:637-42.

28. Broers S, van Vliet KP, le Cessie S, et al. Blood glucose awareness training in Dutch type 1 diabetes patients: one-year follow-up. Neth $J$ Med 2005;63:164-9.

29. Schachinger $\mathrm{H}$, Hegar $\mathrm{K}$, Hermanns $\mathrm{N}$, et al. Randomized controlled clinical trial of Blood Glucose Awareness Training (BGAT III) in Switzerland and Germany. J Behav Med 2005;28:587-94.

30. Pickup JC, Sutton AJ. Severe hypoglycaemia and glycaemic control in Type 1 diabetes: meta-analysis of multiple daily insulin injections compared with continuous subcutaneous insulin infusion. Diabet Med 2008;25:765-74.

31. Pickup J, Mattock M, Kerry S. Glycaemic control with continuous subcutaneous insulin infusion compared with intensive insulin injections in patients with type 1 diabetes: meta-analysis of randomised controlled trials. BMJ 2002;324:705.

32. Ly TT, Nicholas JA, Retterath A, et al. Effect of sensor-augmented insulin pump therapy and automated insulin suspension vs standard insulin pump therapy on hypoglycemia in patients with type 1 diabetes: a randomized clinical trial. JAMA 2013;310:1240-7.

33. Heinemann L, Freckmann G, Ehrmann D, et al. Real-time continuous glucose monitoring in adults with type 1 diabetes and impaired hypoglycaemia awareness or severe hypoglycaemia treated with multiple daily insulin injections (HypoDE): a multicentre, randomised controlled trial. Lancet 2018;391:1367-77.

34. Reddy M, Jugnee N, El Laboudi A, et al. A randomized controlled pilot study of continuous glucose monitoring and flash glucose monitoring in people with Type 1 diabetes and impaired awareness of hypoglycaemia. Diabet Med 2018;35:483-90.

35. Gonder-Frederick L, Shepard J, Peterson N. Closed-loop glucose control: psychological and behavioral considerations. J Diabetes Sci Technol 2011;5:1387-95.

36. Choudhary P, Rickels MR, Senior PA, et al. Evidence-informed clinical practice recommendations for treatment of type 1 diabetes complicated by problematic hypoglycemia. Diabetes Care 2015;38:1016-29.

37. Rogers HA, de Zoysa N, Amiel SA. Patient experience of hypoglycaemia unawareness in Type 1 diabetes: are patients appropriately concerned? Diabet Med 2012;29:321-7.

38. Rankin D, Elliott J, Heller S, et al. Experiences of hypoglycaemia unawareness amongst people with Type 1 diabetes: A qualitative investigation. Chronic IIIn 2014;10:180-91.

39. Anderbro T, Gonder-Frederick L, Bolinder J, et al. Fear of hypoglycemia: relationship to hypoglycemic risk and psychological factors. Acta Diabetol 2015;52:581-9. 
40. Smith $\mathrm{CB}$, Choudhary P, Pernet A, et al. Hypoglycemia unawareness is associated with reduced adherence to therapeutic decisions in patients with type 1 diabetes: evidence from a clinical audit. Diabetes Care 2009;32:1196-8.

41. de Zoysa N, Rogers $\mathrm{H}$, Stadler M, et al. A psychoeducational program to restore hypoglycemia awareness: the DAFNE-HART pilot study. Diabetes Care 2014;37:863-6.

42. Shuttlewood E, De Zoysa N, Rankin D, et al. A qualitative evaluation of DAFNE-HART: a psychoeducational programme to restore hypoglycaemia awareness. Diabetes Res Clin Pract 2015;109:347-54.

43. Heller S, Lawton J, Amiel S, et al. Improving management of type 1 diabetes in the UK: the Dose Adjustment For Normal Eating (DAFNE) programme as a research test-bed. A mixed-method analysis of the barriers to and facilitators of successful diabetes self-management, a health economic analysis, a cluster randomised controlled trial of different models of delivery of an educational intervention and the potential of insulin pumps and additional educator input to improve outcomes. Southampton (UK): NIHR Journals Library, 2014.

44. National Institute for Health and Care Excellence. Type 1 diabetes in adults: diagnosis and management. https://www.nice.org.uk/ guidance/ng17/resources/type-1-diabetes-in-adults-diagnosis-andmanagement-1837276469701 (Accessed 18th Sep 2016).

45. Elliott J, Jacques RM, Kruger J, et al. Substantial reductions in the number of diabetic ketoacidosis and severe hypoglycaemia episodes requiring emergency treatment lead to reduced costs after structured education in adults with Type 1 diabetes. Diabet Med 2014;31:847-53.

46. Gold AE, MacLeod KM, Frier BM. Frequency of severe hypoglycemia in patients with type I diabetes with impaired awareness of hypoglycemia. Diabetes Care 1994;17:697-703.

47. Clarke WL, Cox DJ, Gonder-Frederick LA, et al. Reduced awareness of hypoglycemia in adults with IDDM. A prospective study of hypoglycemic frequency and associated symptoms. Diabetes Care 1995; 18:517-22.

48. Gadsby R, Snow R, Daly AC, et al. Setting research priorities for Type 1 diabetes. Diabet Med 2012;29:1321-6.

49. Margiotta G, Smith E, Halevy C, et al. Differences in cognitions and behaviours between adults with type 1 diabetes with intact and impaired awareness of hypoglycaemia. Diabetologia 2016. Abstract 84.

50. Gonder-Frederick LA, Schmidt KM, Vajda KA, et al. Psychometric properties of the hypoglycemia fear survey-ii for adults with type 1 diabetes. Diabetes Care 2011;34:801-6.

51. Singh H, Gonder-Frederisk L, Schmidt K, et al. Assessing hyperglycemia avoidance in people with type 1 diabetes. Diabetes Management 2014;4:263-71.
52. Polonsky WH, Anderson BJ, Lohrer PA, et al. Assessment of diabetes-related distress. Diabetes Care 1995;18:754-60.

53. Zigmond AS, Snaith RP. The hospital anxiety and depression scale. Acta Psychiatr Scand 1983;67:361-70.

54. Speight J, Barendse SM, Singh H, et al. Characterizing problematic hypoglycaemia: iterative design and preliminary psychometric validation of the Hypoglycaemia Awareness Questionnaire (HypoA-Q). Diabet Med 2016;33:376-85.

55. Cooke D, O'Hara MC, Beinart N, et al. Linguistic and psychometric validation of the Diabetes-Specific Quality-of-Life Scale in U.K. English for adults with type 1 diabetes. Diabetes Care 2013;36:1117-25.

56. Dahlem NW, Zimet GD, Walker RR. The multidimensional scale of perceived social support: a confirmation study. J Clin Psychol 1991;47:756-61.

57. Wilkinson G, Drummond M. Alternative approaches for assessing the socioeconomic benefits of medical devices: a systematic review. Expert Rev Med Devices 2015;12:629-48.

58. Thornicroft G. An adapted version of the Client Service Receipt Inventory (CSRI; Beecham J \& Knapp M. 2001. Costing psychiatric interventions. Measuring mental health needs. 2nd edn. Gaskell, London.

59. Medical Research Council. Developing and evaluating complex interventions: new guidance. 2006 https://www.mrc.ac.uk/ documents/pdf/complex-interventions-guidance/.

60. Curran GM, Bauer M, Mittman B, et al. Effectiveness-implementation hybrid designs: combining elements of clinical effectiveness and implementation research to enhance public health impact. Med Care 2012;50:217-26.

61. Soukup T, Hull L, Smith EL, et al. An effectiveness-implementation hybrid type 2 trial evaluating two educational programmes targeting severe hypoglycaemia in people with type 1 diabetes: Implementation study protocol. BMJ Open 2019. under review.

62. Wagner CC, Ingersoll KS. Development and initial validation of the assessment of motivational interviewing groups - observer scales (AMIGOS). Int J of Group Psychotherapy 2018;68:69-79.

63. Baldwin SA, Murray DM, Shadish WR, et al. Intraclass correlation associated with therapists: estimates and applications in planning psychotherapy research. Cogn Behav Ther 2011;40:15-33.

64. UK Hypoglycaemia Study Group. Risk of hypoglycaemia in types 1 and 2 diabetes: effects of treatment modalities and their duration. Diabetologia 2007;50:1140-7.

65. Pedersen-Bjergaard U, Færch L, Allingbjerg ML, et al. The influence of new European Union driver's license legislation on reporting of severe hypoglycemia by patients with type 1 diabetes. Diabetes Care 2015;38:29-33. 